\title{
Predicting Nonlinear Stiffness, Motion Range, and Load-Bearing Capability of Leaf-Type Isosceles-Trapezoidal Flexural Pivot Using Comprehensive Elliptic Integral Solution
}

\author{
Aimei Zhang $(\mathbb{D}$, Yanjie Gou, and Xihui Yang \\ School of Electro-Mechanical Engineering, Xidian University, Xi'an, Shaanxi 710071, China \\ Correspondence should be addressed to Aimei Zhang; aimeizh@126.com
}

Received 19 November 2019; Accepted 16 January 2020; Published 12 February 2020

Academic Editor: Hervé Laurent

Copyright (c) 2020 Aimei Zhang et al. This is an open access article distributed under the Creative Commons Attribution License, which permits unrestricted use, distribution, and reproduction in any medium, provided the original work is properly cited.

\begin{abstract}
A leaf-type isosceles-trapezoidal flexural (LITF) pivot consists of two leaf springs that are situated in the same plane and intersect at a virtual center of motion outside the pivot. The LITF pivot offers many advantages, including large rotation range and monolithic structure. Each leaf spring of a LITF pivot subject to end loads is deflected into an S-shaped configuration carrying one or two inflection points, which is quite difficult to model. The kinetostatic characteristics of the LITF pivot are precisely modeled using the comprehensive elliptic integral solution for the large-deflection problem derived in our previous work, and the strengthchecking method is further presented. Two cases are employed to verify the accuracy of the model. The deflected shapes and nonlinear stiffness characteristics within the range of the yield strength are discussed. The load-bearing capability and motion range of the pivot are proposed. The nonlinear finite element results validate the effectiveness and accuracy of the proposed model for LITF pivots.
\end{abstract}

\section{Introduction}

A leaf-type isosceles-trapezoidal flexural (LITF) pivot consists of two leaf springs located in the same plane [1]. The two leaf springs are arranged symmetrically and intersect at a virtual center of motion outside the pivot, as shown in Figure 1. The parallelogram flexure is a special type in which the two leaf springs intersect at infinity. LITF pivots have been utilized in many accurate mechanisms [2-4] due to their obvious advantages such as low cost, monolithic manufacturing, reduced weight, and smooth motion [5-7].

When delivering movement, the leaf springs of a LITF pivot undergo nonlinear large deflection that may carry one or two inflection points (where the resultant moment is equal to zero [8]), which complicates the accurate modeling of LITF pivots. The remote center location and stiffness of a LITF pivot are presented by the model of screw theory based on the small-deflection assumption [9], which limits the application range of the model. The two pseudorigid-body models with small-deflection assumption [10], i.e., a four- bar model and a pin-joint model, were proposed for the analysis of the moment-angle characteristics of LITF pivots subject to horizontal force and moment. However, the influence of vertical force to LITF pivots was neglected. The analytic models for stiffness and center shift were presented by using the beam constraint model (BCM) method [11], which can be used to solve the nonlinear characteristics of LITF pivots, i.e., the rotation angle is in the range of $\pm 15^{\circ}$. The efficiency of uniform-strength composite leaf springs under various loading conditions [12] was analyzed. Therefore, the accurate nonlinear analysis and load-bearing capability solution within the entire stress range are indispensable for the application of LITF pivots.

Because the leaf spring is so thin and flexible that the effects on axial elongation and shear are negligible, the elliptic integral solution is often considered to be the most accurate model for modeling this kind of large deflection beams. Howell [13] presented the elliptic integral solutions for the large deflection beam with no inflection point. An elliptic integral solution for the beam with an inflection 


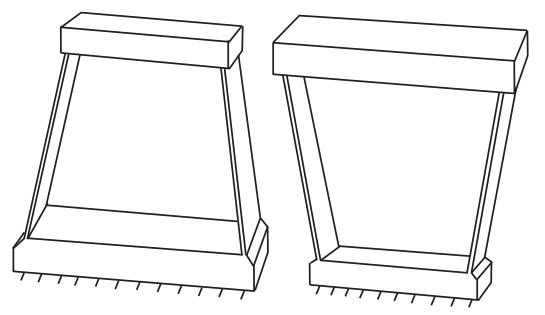

(a)

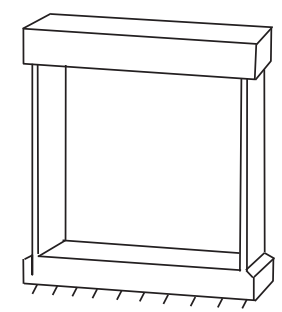

(b)

FIgURE 1: Classification of the leaf-type isosceles-trapezoidal flexural (LITF) pivot (a) and parallelogram flexure (b).

point was derived by Kimball and Tsai [14]. In our previous research [15], we developed the comprehensive elliptic integral solution to solve the large deflections of beams with multiple inflection points and subject to any kinds of load cases. Because each of the deflected leaf spring carry one or two inflection points, LITF pivots can be modeled by the comprehensive elliptic integral solution. The model can be used to solve the exact deflected shapes and nonlinear stiffness of LITF pivots subject to different loads. Through the stress analysis for deflected leaf springs, the maximum motion range and allowable loads of LITF pivots are solved.

The rest of this paper is organized as follows. In Section 2 , the accurate kinetostatic model and stress check for LITF pivots are proposed. In Section 3, two examples are calculated to demonstrate the accuracy of the model for LITF pivots. The nonlinear stiffness and workspace evaluation of the two examples are then discussed. In Section 4, concluding remarks are presented.

\section{Modeling}

2.1. LITF Pivot. As shown in Figure 2, two springs $\left(O_{1} A\right.$ and $\mathrm{O}_{2} A$, length $L$ ) of a LITF pivot intersect at point $O \prime$ and the angle between two leaf springs is $2 \beta$. The lengths of $\mathrm{O}_{1} \mathrm{O}_{2}$ and $A B$ are $w_{1}$ and $w_{2}$, respectively. Letting $N=w_{2} / w_{1}$, when $N \neq 1$, we then have

$$
\left.\begin{array}{l}
w_{1}=\frac{2 L \sin \beta}{|N-1|} \\
w_{2}=\frac{2 N L \sin \beta}{|N-1|}
\end{array}\right\} .
$$

When $N=1$, the LITF pivot becomes a parallelogram flexure, as shown in Figure 2(c), $\beta=0^{\circ}$ and $w_{1}=w_{2}$.

The global coordinate system OXY is established for the LITF pivot with the $X$ axis oriented along $\mathrm{O}_{2} \mathrm{O}_{1}$ and the origin located at the midpoint of $O_{2} O_{1}$, as shown in Figure 2. The initial angle between leaf spring $O_{1} A$ and the $X$ axis is $\theta_{1}$, and the angle between leaf spring $O_{2} B$ and the $X$ axis is $\theta_{2}$. For $N<1, \theta_{1}=90^{\circ}+\beta$ and $\theta_{2}=90^{\circ}-\beta$. For $N>1, \theta_{1}=$ $90^{\circ}-\beta$ and $\theta_{2}=90^{\circ}+\beta$.

The local coordinate systems $O_{1} X_{1} Y_{1}$ and $O_{2} X_{2} Y_{2}$ for leaf springs $O_{1} A$ and $O_{2} B$ are established with the origins placed at the fixed end and the $X_{1}$ and $X_{2}$ axes oriented along the leaf springs, respectively. The deflected end coordinates and the end angle of spring $O_{1} A$ with respect to the local coordinate $O_{1} X_{1} Y_{1}$ are $a_{1}, b_{1}$, and $\theta_{1 o}$, respectively. Similarly, the corresponding end coordinates and angle of $\mathrm{O}_{2} B$ with respect to the local coordinate $\mathrm{O}_{2} X_{2} Y_{2}$ are denoted $a_{2}, b_{2}$, and $\theta_{20}$, respectively. The horizontal displacement $\Delta X$, vertical displacement $\Delta Y$, and rotation angle $\Delta \theta$ of the freedom for the LITF pivot in the global coordinate system are expressed as

$$
\left.\begin{array}{l}
\Delta \theta=\theta_{1 o}=\theta_{2 o} \\
\Delta X=\frac{1}{2}\left[\left(a_{1} \cos \theta_{1}+a_{2} \cos \theta_{2}\right)-\left(b_{1} \sin \theta_{1}+b_{2} \sin \theta_{2}\right)\right] \\
\Delta Y=\frac{1}{2}\left[\left(a_{1} \sin \theta_{1}+a_{2} \sin \theta_{2}\right)+\left(b_{1} \cos \theta_{1}+b_{2} \cos \theta_{2}\right)\right]-L \cos \beta
\end{array}\right\}
$$

The loop closure equations are given as

$$
\begin{aligned}
{\left[\begin{array}{cc}
\cos \theta_{1} & -\sin \theta_{1} \\
\sin \theta_{1} & \cos \theta_{1}
\end{array}\right]\left\{\begin{array}{l}
a_{1} \\
b_{1}
\end{array}\right\}=} & {\left[\begin{array}{cc}
\cos \theta_{2} & -\sin \theta_{2} \\
\sin \theta_{2} & \cos \theta_{2}
\end{array}\right]\left\{\begin{array}{l}
a_{2} \\
b_{2}
\end{array}\right\}-\left\{\begin{array}{c}
w_{1} \\
0
\end{array}\right\} } \\
& +w_{2}\left\{\begin{array}{c}
\cos \Delta \theta \\
\sin \Delta \theta
\end{array}\right\} .
\end{aligned}
$$

Figure 3 shows the free-body diagrams for link $\mathrm{AB}$ and leaf springs $O_{1} A$ and $O_{2} B$. When the pivot is subject to horizontal force $F_{x}$, vertical force $F_{y}$, and moment $M$ at the midpoint $C$ of link $A B$, the horizontal and vertical components of the end force and moment loaded at the $O_{1} A$ and $O_{2} B$ are $F_{1 x}, F_{1 y}, M_{1 o}$ and $F_{2 x}, F_{2 y}, M_{2 o}$, respectively. Applying the static equilibrium for link $A B$ yields

$$
\left.\begin{array}{l}
F_{1 x}+F_{2 x}+F_{x}=0 \\
-F_{1 y}-F_{2 y}+F_{y}=0 \\
-M_{1 o}-M_{2 o}+M-F_{1 x} w_{2} \sin \Delta \theta-F_{1 y} w_{2} \cos \Delta \theta \\
-\frac{F_{x} w_{2} \sin \Delta \theta}{2}+\frac{F_{y} w_{2} \cos \Delta \theta}{2}=0
\end{array}\right\} .
$$

$P_{1}$ and $n_{1} P_{1}$ are the components of $F_{1 x}$ and $F_{1 y}$ along $O_{1} Y_{1}$ and $X_{1} O_{1}$ and have

$$
\left\{\begin{array}{l}
F_{1 x} \\
F_{1 y}
\end{array}\right\}=\left[\begin{array}{cc}
\cos \theta_{1} & \sin \theta_{1} \\
-\sin \theta_{1} & \cos \theta_{1}
\end{array}\right]\left\{\begin{array}{c}
n_{1} P_{1} \\
P_{1}
\end{array}\right\} .
$$

Similarly, for $\mathrm{O}_{2} B$, 


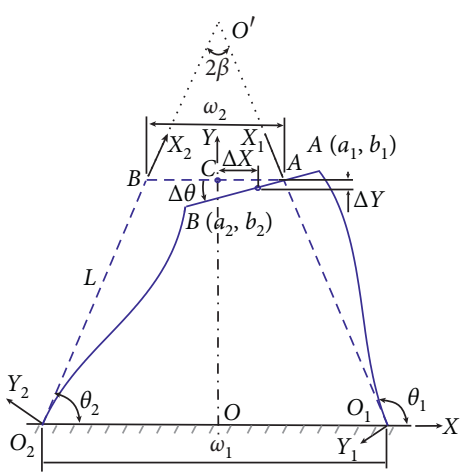

(a)

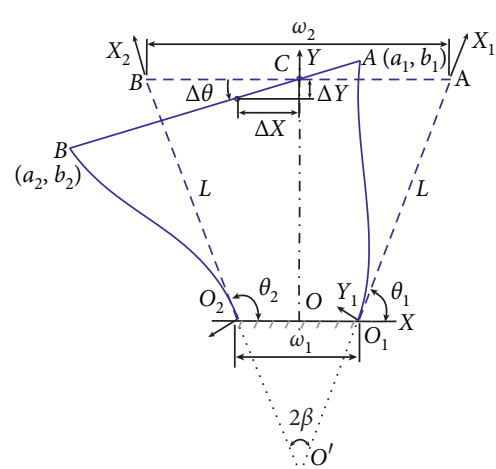

(b)

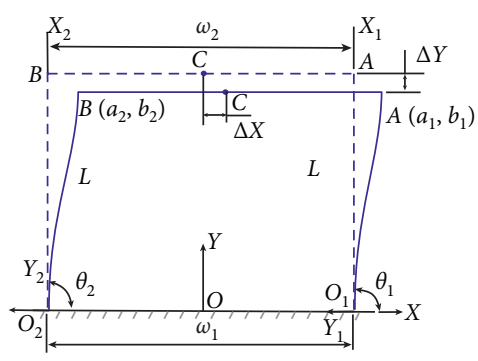

(c)

Figure 2: Deformation diagram of LITF pivot. (a) $N<1$. (b) $N>1$. (c) $N=1$.

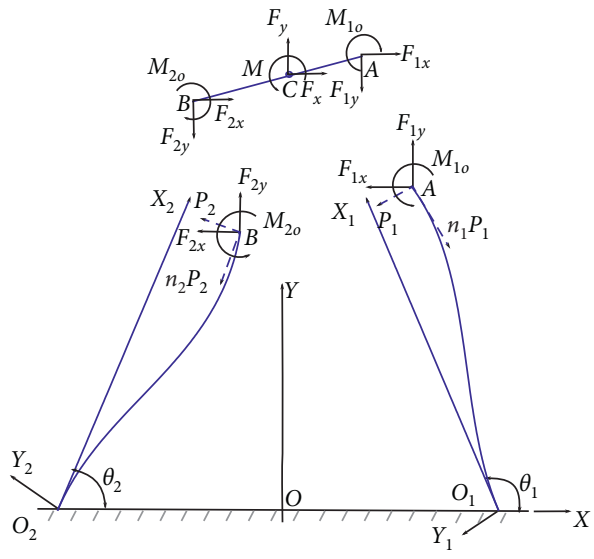

(a)

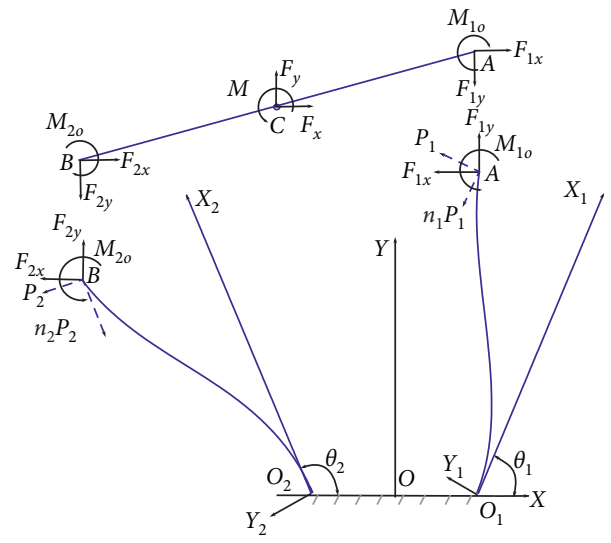

(b)

FIGURE 3: Force analysis of the LITF pivot. (a) $N \leq 1$ and (b) $N>1$.

$$
\left\{\begin{array}{c}
F_{2 x} \\
F_{2 y}
\end{array}\right\}=\left[\begin{array}{cc}
\cos \theta_{2} & \sin \theta_{2} \\
-\sin \theta_{2} & \cos \theta_{2}
\end{array}\right]\left\{\begin{array}{c}
n_{2} P_{2} \\
P_{2}
\end{array}\right\} .
$$

The deflection of each leaf spring can be modeled using the comprehensive elliptic integral solution summarized in the following section. The comprehensive elliptic integral solution for each leaf spring, together with the loop closure equation equation (3) and the static equilibrium equation (4), constitute the kinetostatic model for LITF pivots.

2.2. Comprehensive Elliptic Integral Solution. Each of the two springs of a LITF pivot can be viewed as a cantilever beam subject to an end vertical force $P$, an end horizontal force $n P$, and an end moment $M_{o}$, as shown in Figure 4. The tip coordinates and tip angle of the deflected beam are denoted as $a, b$, and $\theta_{o}$, respectively. Each deflected spring may carry $m$ inflection points $(m=1$ or 2$)$. The comprehensive solution [15] for the beam with inflection points, as summarized in the following, formulates the load parameters $(\kappa$, $n$, and $\alpha$ ) and deflection parameters $\left(a, b\right.$, and $\left.\theta_{o}\right)$ by introducing $m$ as the shape parameter:

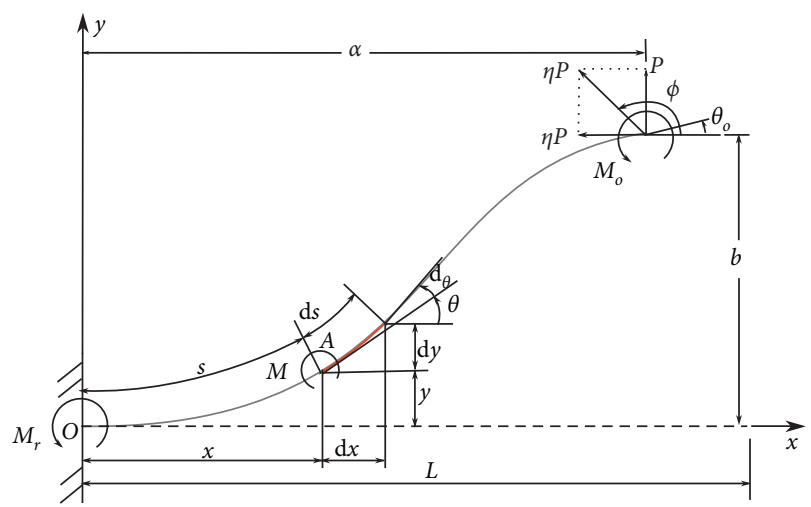

Figure 4: Cantilever beam subject to combined force and moment loads.

$$
\left.\begin{array}{l}
\alpha=\frac{S_{r}}{\sqrt{\eta}} f \\
\frac{a}{L}=\frac{S_{r}}{\alpha \eta^{5 / 2}}[-n \eta f+2 n \eta e+\sqrt{2 \eta} c] \\
\frac{b}{L}=\frac{S_{r}}{\alpha \eta^{5 / 2}}[\eta f-2 \eta e+n \sqrt{2 \eta} c]
\end{array}\right\},
$$


where $\alpha$ is defined as the force index (EI is the flexural rigidity of the beam),

$$
\alpha=\sqrt{\frac{\mathrm{PL}^{2}}{\mathrm{EI}}}
$$

$\kappa$ is the load ratio,

$$
\kappa=\frac{M_{o}^{2}}{2 \mathrm{PEI}}
$$

and $S_{r}$ is the sign of the resulting moment at the fixed end of the beam,

$$
S_{r}= \begin{cases}(-1)^{m}, & M_{o} \geq 0 \\ (-1)^{m+1}, & M_{0}<0 .\end{cases}
$$

Moreover,

$$
\begin{aligned}
& \lambda=\sin \theta_{o}-n \cos \theta_{o}+\kappa, \\
& \eta=\sqrt{1+n^{2}}, \\
& f=(-1)^{m} F\left(\gamma_{2}, t\right)-F\left(\gamma_{1}, t\right)+2 m S_{r} F(t), \\
& e=(-1)^{m} E\left(\gamma_{2}, t\right)-E\left(\gamma_{1}, t\right)+2 m S_{r} E(t), \\
& \gamma_{1}=\sin ^{-1} \sqrt{\frac{\eta-n}{\lambda+\eta}} \\
& \gamma_{2}=\left\{\begin{array}{l}
\sin ^{-1} \sqrt{\frac{\eta+\sin \theta_{o}-n \cos \theta_{o}}{\lambda+\eta}}-\pi+\phi<\theta_{o} \leq \phi, \\
-\sin ^{-1} \sqrt{\frac{\eta+\sin \theta_{o}-n \cos \theta_{o}}{\lambda+\eta}}-2 \pi+\phi<\theta_{o} \leq-\pi+\phi,
\end{array}\right. \\
& t=\sqrt{\frac{\lambda+\eta}{2 \eta}} \\
& c=\sqrt{\lambda+n}-(-1)^{m} \sqrt{\lambda-\sin \theta_{o}+n \cos \theta_{o}} .
\end{aligned}
$$

$F(\gamma, t)$ and $E(\gamma, t)=\int_{0}^{\gamma} \sqrt{1-t \sin \delta \mathrm{d} \delta}$ are the incomplete elliptic integrals of the first and second kinds [16], respectively, in which $\gamma$ is called the amplitude and $t(-1 \leq t \leq 1)$ is the modulus. When $\gamma=\pi / 2$, they become the complete elliptic integrals of the first and second kinds and are denoted as $F(t)$ and $E(t)$, respectively. $\phi$ represents the angle of the force applied at the free end (as marked in Figure 4):

$$
\phi=\frac{\pi}{2}+\arctan n \text {. }
$$

The coordinates $(x, y)$ of an arbitrary point $A$ on the beam (shown in Figure 4) can be written as

$$
\left.\begin{array}{l}
\frac{x}{L}=\frac{S_{r}}{\alpha \eta^{5 / 2}}[-n \eta f(\theta)+2 n \eta e(\theta)+\sqrt{2 \eta} c(\theta)] \\
\frac{y}{L}=\frac{S_{r}}{\alpha \eta^{5 / 2}}[\eta f(\theta)-2 \eta e(\theta)+n \sqrt{2 \eta} c(\theta)]
\end{array}\right\}
$$

where $\theta$ is the deflected angle at point $A$ and

$$
\begin{aligned}
& \begin{aligned}
& f(\theta)=(-1)^{m(\theta)} F(\gamma, t)-F\left(\gamma_{1}, t\right)+2 m(\theta) S_{r} F(t), \\
& e(\theta)=(-1)^{m(\theta)} E(\gamma, t)-E\left(\gamma_{1}, t\right)+2 m(\theta) S_{r} E(t), \\
& \gamma=\left\{\begin{array}{l}
\sin ^{-1} \sqrt{\frac{\eta+\sin \theta-n \cos \theta}{\lambda+\eta}}-\pi+\phi<\theta \leq \phi, \\
-\sin ^{-1} \sqrt{\frac{\eta+\sin \theta-n \cos \theta}{\lambda+\eta}}-2 \pi+\phi<\theta \leq-\pi+\phi,
\end{array}\right. \\
& c(\theta)=\sqrt{\lambda+n}-(-1)^{m(\theta)} \sqrt{\lambda-\sin \theta+n \cos \theta} .
\end{aligned}
\end{aligned}
$$

The value $m(\theta)$ is equal to the number of the inflection points between the fixed end and point $A$.

Most deflected springs mainly loaded by the horizontal force $F_{x}$ and the moment $M$ have one inflection point $(m=1)$. When the vertical force dominates in the applied loads, the deflected spring may contain two inflection points $(m=2)$.

The kinetostatic model applied for LITF pivots can be calculated by the built-in function "fsolve" in MatLab (MathWorks, USA). The deflected shapes and the nonlinear stiffness characteristics of LITF pivots are further shown in Section 3. However, the stress of deflected leaf springs should be less than the maximum yield stress, which determines the motion range and load-bearing capacity of LITF pivots.

2.3. Stress Analysis. The maximum bending stress, the important index of checking the LITF pivot, decides the maximum rotation angle and the maximum allowable loads. For deflected LITF pivots, the maximum bending stress $\sigma_{\max }$ of each spring should be less than the yield strength $S_{y}$ of the material used; thus, for the rectangular cross section $I=$ $w h^{3} / 12$ ( $w$ is the width and $h$ is the height), we have [17]

$$
\sigma_{\max }=\frac{h|M|_{\max }}{2 I} \leq S_{y}
$$

where $|M|_{\max }$ is the maximum resultant moment distributing in leaf springs. Substituting curvature $K=M / E I$ into equation (15) yields

$$
\sigma_{\max }=\frac{\mathrm{Eh}|K|_{\max }}{2} \leq S_{y}
$$

where $|K|_{\max }$ is the maximum curvature occurring in the two deflected leaf springs.

The motion range and load-bearing capability of LITF pivots subject to different loads, as two of the most important criteria with which to compare the compliant joints, can be obtained by the kinetostatic model and stresschecking equation (16).

The flowchart of the solution to the deflected shape and workspace evaluation of LITF pivots is shown in Figure 5. 


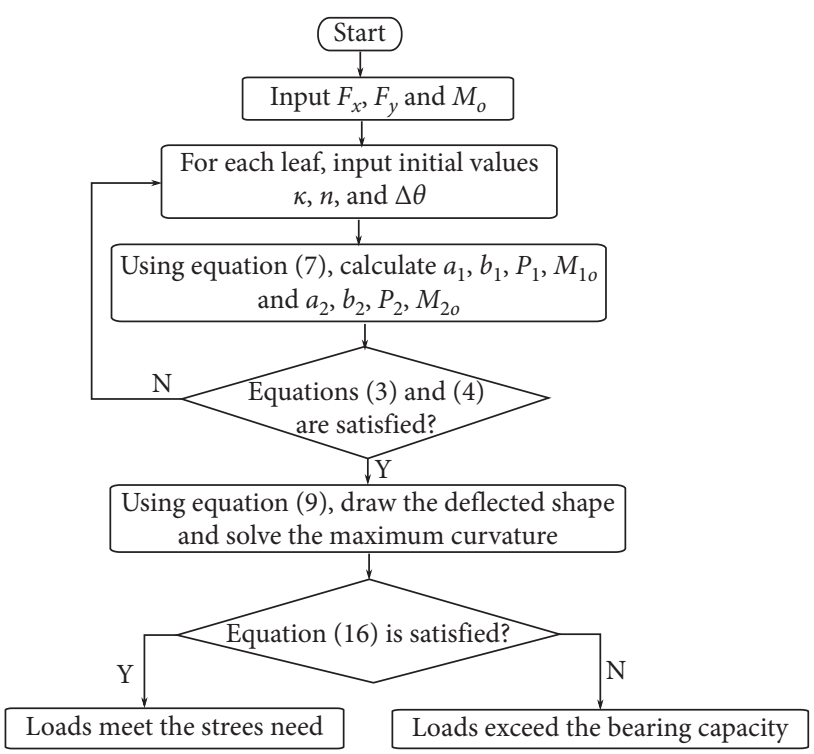

FIGURE 5: The flowchart of the solution to LITF pivots.

\section{Case Studies}

In this section, a LITF pivot and a parallelogram flexure are employed as two cases to demonstrate the effectiveness of the comprehensive elliptic integral model. The parameters of the two pivots are given in Table 1 , and the materials are polypropylene in which $E=1.4 \mathrm{GPa}$ and $S_{y}=34 \mathrm{MPa}$ [13].

3.1. Solution for LITF Pivot. The parameters of the LITF pivot are shown in Table 1. The lengths of link $O_{1} O_{2}$ and $A B$ solved by equation (1) are $w_{1}=0.1167 \mathrm{~m}$ and $w_{2}=0.0467 \mathrm{~m}$, respectively. The deflected shapes of the pivot subject to different loads, the load-bearing capacity, and the corresponding motion range of the pivot will be discussed here.

3.1.1. Deflected Shapes under Different Loads. The deflected results of the pivot subject to different loads are obtained separately by using the comprehensive elliptic integral solution and a nonlinear finite element analysis (NFEA) model, as shown in Figures 6-10. For the NFEA model built with the ANSYS software, springs $O_{1} A$ and $O_{2} B$ are meshed into 100 elements with BEAM188, respectively, and the large displacement analysis option is turned on. BEAM188 is suitable for analyzing slender to moderately stubby beam structures. This element is based on the Timoshenko beam theory. Shear deformation is included. The results of the comprehensive elliptic integral solution agree well with NFEA.

For the LITF pivot subject to pure moment loaded at point $\mathrm{C}$, the relationship between the rotation angle $\Delta \theta$ and the moment $M$ is shown in Figure 6. The LITF pivot reveals fine linearity for $\Delta \theta$ less than $10^{\circ}$ (the dashed line in Figure 6 expresses the linear approximation of the LITF pivot with small deformation). However, when $\Delta \theta$ is larger than $10^{\circ}$, the nonlinearity of the stiffness for this kind of pivot
TABLE 1: Parameters of pivot.

\begin{tabular}{lccccccc}
\hline & $w(\mathrm{~m})$ & $h(\mathrm{~m})$ & $L(\mathrm{~m})$ & $N$ & $\beta\left(^{\circ}\right)$ & $w_{1}(\mathrm{~m})$ & $w_{2}(\mathrm{~m})$ \\
\hline $\begin{array}{l}\text { LITF pivot } \\
\text { Parallelogram } \\
\text { flexure }\end{array}$ & 0.0252 & 0.001 & 0.07 & 0.4 & $30^{\circ}$ & - & - \\
& 0.0252 & 0.001 & 0.07 & 1 & $0^{\circ}$ & 0.1 & 0.1 \\
\hline
\end{tabular}

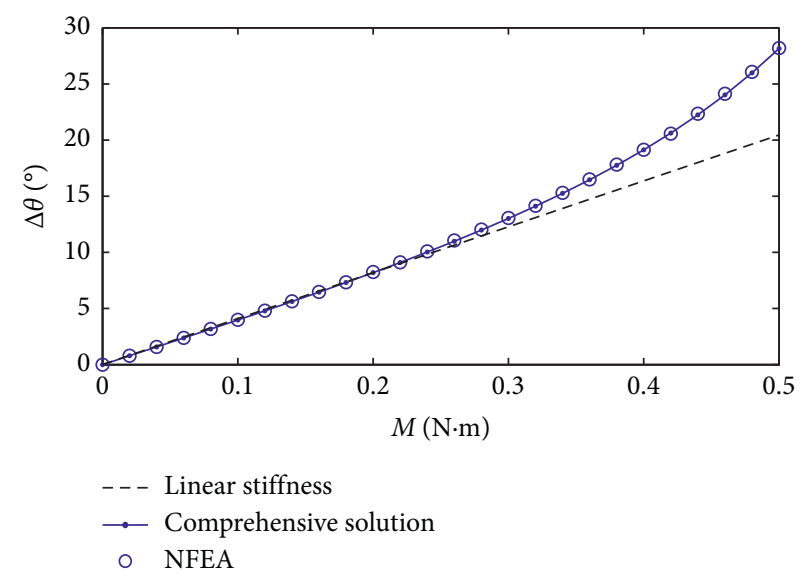

Figure 6: Plots of moment $M$ vs angle $\Delta \theta$.

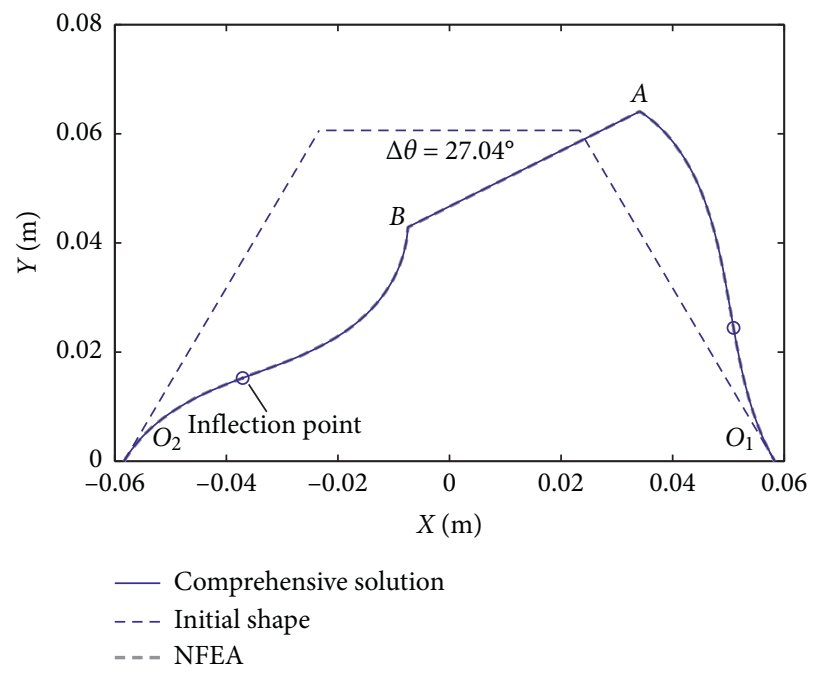

Figure 7: Deflected shape of positive pivot subject to $F_{x}=0 \mathrm{~N}$, $F_{y}=0 \mathrm{~N}$, and $M=0.49 \mathrm{~N} \cdot \mathrm{m}$.

becomes remarkable. For $M=0.49 \mathrm{~N} \cdot \mathrm{m}$, the maximum curvature of the pivot occurring at point $\mathrm{A}$ is equal to $48.5519 \cdot \mathrm{m}^{-1}$, which is substituted into equation (16) to obtain the maximum stress, $\sigma_{\max }=33.986 \mathrm{MPa}$, close to the yield strength. Meanwhile, $\Delta \theta$ attains $27.04^{\circ}$, for which the deflected shape of the pivot is shown in Figure 7.

If $F_{x}=5 \mathrm{~N}$ and $M$ are loaded at point $\mathrm{C}$ simultaneously, the relationships of $\Delta X, \Delta Y$, and $\Delta \theta$ with $M$ before yield failure have slight nonlinearities, as shown in Figures 8-10. Otherwise, for $F_{x}=-5 \mathrm{~N}, F_{y}=0 \mathrm{~N}$, and different $M$, the nonlinearities of the pivot become obvious. For $M$ from 0 to 


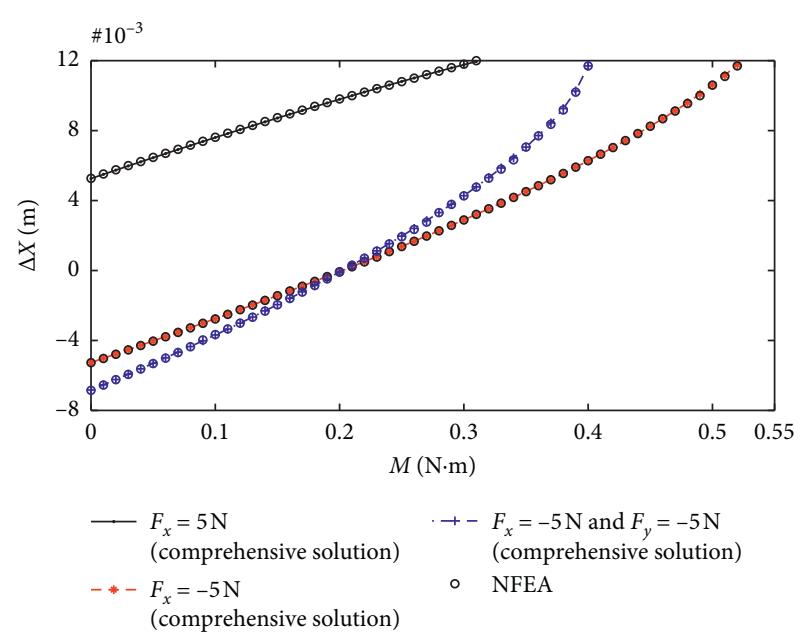

Figure 8: Plots of moment $M$ vs horizontal displacement $\Delta X$.

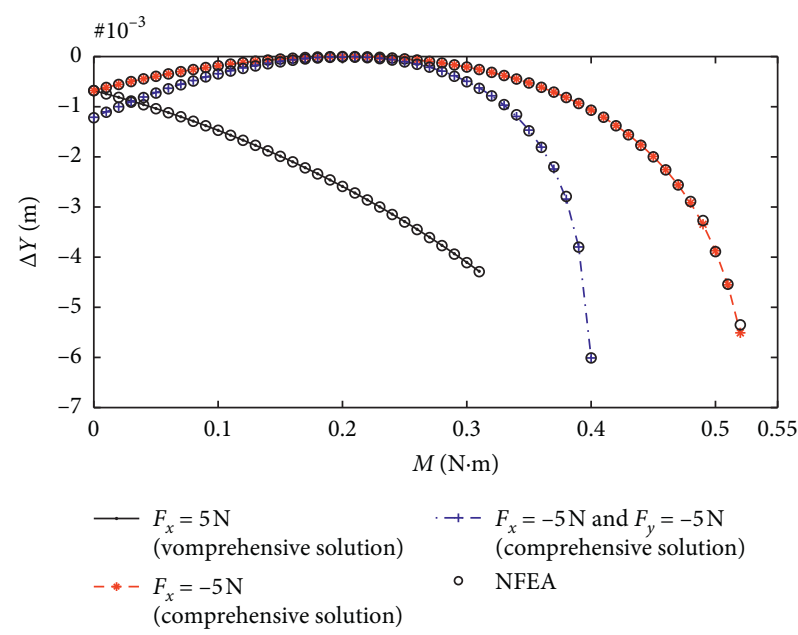

Figure 9: Plots of moment $M$ vs vertical displacement $\Delta Y$.

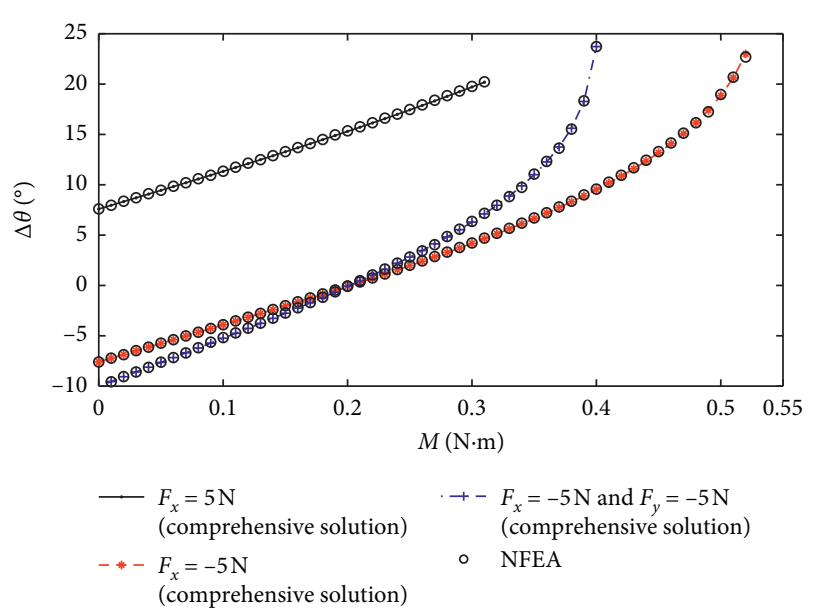

Figure 10: Plots of moment $M$ vs angle $\Delta \theta$.

$0.5 \mathrm{~N} \cdot \mathrm{m}, \Delta X$ varies from $-0.005 \mathrm{~m} \mathrm{~m}$ to $0.011 \mathrm{~m}$ and $\Delta Y$ increases from $-0.0007 \mathrm{~m}$ to $0 \mathrm{~m}$ and then decreases to $-0.004 \mathrm{~m}$ again and $\Delta \theta$ changes from $-7.6^{\circ}$ to $18.9^{\circ}$, as shown in Figures 8-10. The curve of $F_{x}=-5 \mathrm{~N}$ and $F_{y}=0 \mathrm{~N}$ is intersected at $M=0.2$ with that of $F_{x}=-5$ and $F_{y}=-5$, where the resultant moments for the rotation center $O \prime$ are equal to zero, so that the pivot returns to the original position. The corresponding deflected shapes of the pivot for $F_{x}=-5 \mathrm{~N}, F_{y}=0 \mathrm{~N}$, and $M=0 \sim 0.5 \mathrm{~N} \cdot \mathrm{m}$, as shown in Figure 11, incline to the left and then to the right.

3.1.2. Workspace Evaluation. The stress of the deflected pivot solved by the kinetostatic model is checked by equation (16), and then the load-bearing capacity in different load cases and the motion range of the pivot are obtained.

(1) Horizontal Force and Moment. Figure 12 shows that the pivot subject to different $M$ and $F_{x}$ can bear a range of horizontal force. The arrows drawn in Figure 12 roughly mark the descending direction of the stress, and the covered area is the safe working region.

The pivot only subjected to horizontal force, i.e., $M=0$, can bear the maximum horizontal force reaching $F_{x}= \pm 13.6 \mathrm{~N}$, for which the corresponding rotation angles are $\Delta \theta= \pm 16.9^{\circ}$, as shown in Figure 13. With the incremental moment, the maximum positive horizontal force of the pivot gradually decreases and the anticlockwise rotation angle of link $\mathrm{AB}$ shows the increasing tendency.

For $M=0.49 \mathrm{~N} \cdot \mathrm{m}$, the maximum stress of the pivot without horizontal force reaches the yield strength. When negative horizontal force and moment act on the pivot, the negative allowable horizontal force increases gradually and the corresponding angle decreases slightly with the increasing moment, as shown in Figures 12 and 13. For $M=0.5 \mathrm{~N} \cdot \mathrm{m}$, the allowable negative force is $F_{x}=-24.12 \mathrm{~N}$ and $\Delta \theta=-13.06^{\circ}$, as shown in Figure 14, and the maximum curvature also happens at point $\mathrm{A}$.

The relative errors of the rotation angles between the comprehensive solution $\left(\Delta \theta_{\mathrm{CS}}\right)$ and the nonlinear finite element $\left(\Delta \theta_{\mathrm{FEA}}\right)$ results are expressed as

$$
\text { Error }=\frac{\Delta \theta_{\mathrm{CS}}}{\Delta \theta_{\mathrm{FEA}}}-1 .
$$

The errors of the positive rotation angles depicted in Figure 13 between the comprehensive solution and the nonlinear finite element results are less than $1.5 \%$, which is shown in Figure 15.

(2) Vertical Force and Moment. For the pivot subject to $M$ and $F_{y}$, Figure 16 draws the maximum vertical force that the pivot subject to different moments can bear. Similarly, the declining direction of the stress is masked roughly by the arrows in Figure 16. Positive vertical force can counteract the rotation angle of the pivot caused by the moment. It should be noted that the tensile stress might lead to the failure of the pivot when positive vertical force reaches a certain value because the Bernoulli-Euler beam theory neglects the effect of axial elongation and the maximum positive vertical force cannot be predicted, the discussion of which is outside the scope of this paper. 


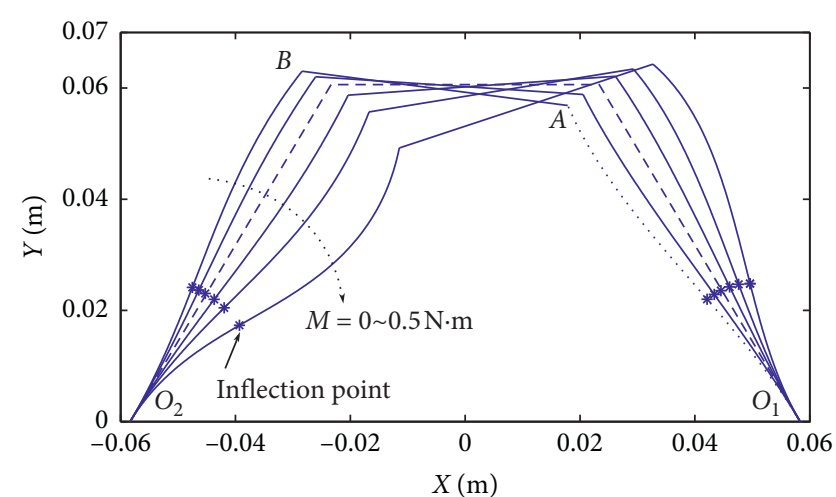

FIgURE 11: Deflected shapes of the positive pivot subject to $F_{x}=-5 \mathrm{~N}, F_{y}=0 \mathrm{~N}$, and $M=0 \sim 0.5 \mathrm{~N} \cdot \mathrm{m}$.

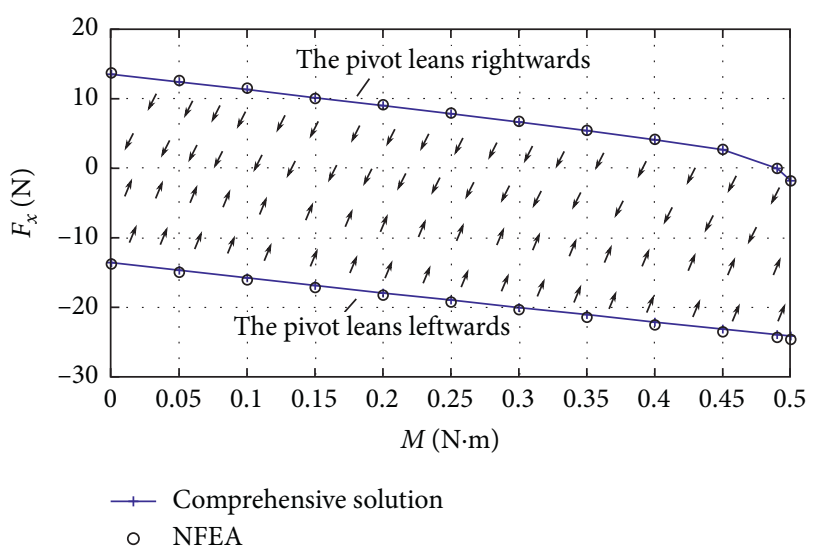

FIgURE 12: Allowable maximum horizontal force of the pivot subject to different moments.

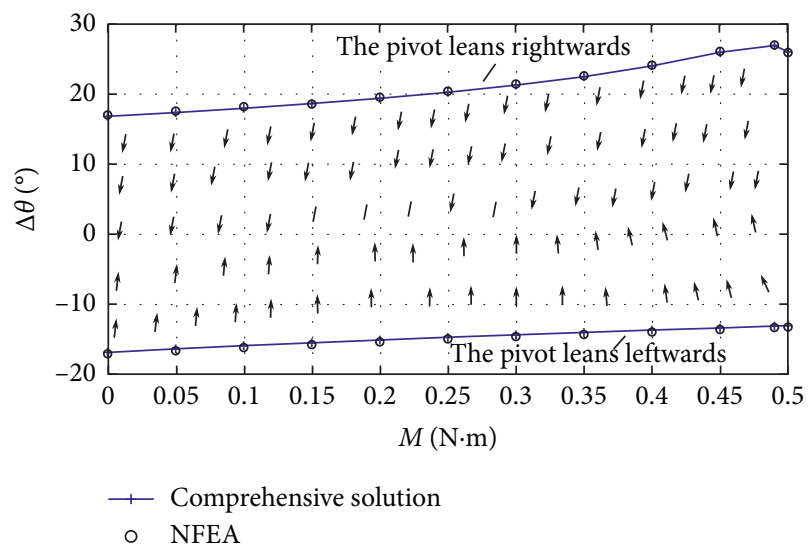

FIGURE 13: Corresponding rotation angle of the pivot subject to different moments and maximum horizontal force depicted in Figure 12.

The corresponding rotation angles subject to different moments and the maximum vertical forces depicted in Figure 16 are shown in Figure 17. The rotation angle slightly increases for $M<0.2 \mathrm{~N} \cdot \mathrm{m}$ and then decreases for $M>0.2 \mathrm{~N} \cdot \mathrm{m}$. When $M=0.2 \mathrm{~N} \cdot \mathrm{m}$, the allowable negative

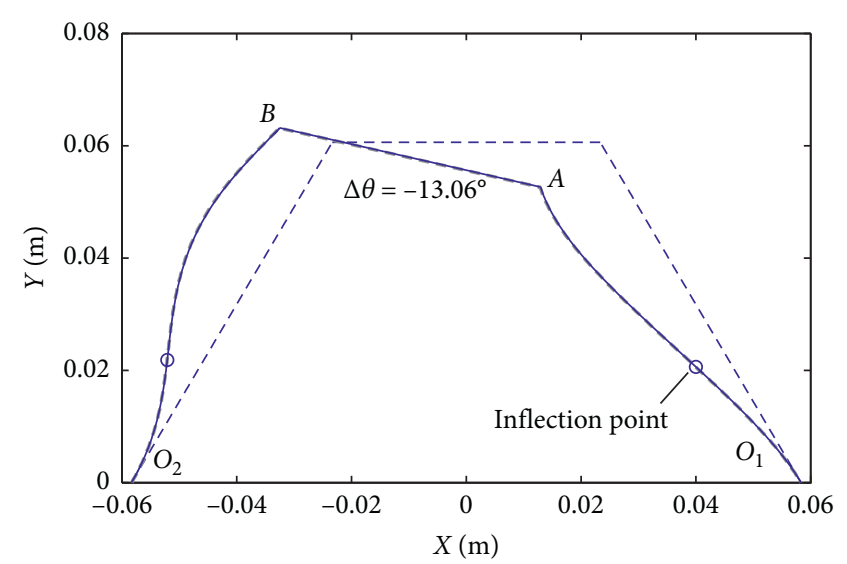

$$
\begin{aligned}
& \text { - Comprehensive solution } \\
& \text { - - Initial shape } \\
& \text { - - - NFEA }
\end{aligned}
$$

FIgURE 14: Deformed shape of the positive pivot subject to $F_{x}=-24.21 \mathrm{~N}, F_{y}=0 \mathrm{~N}$, and $M=0.5 \mathrm{~N} \cdot \mathrm{m}$.

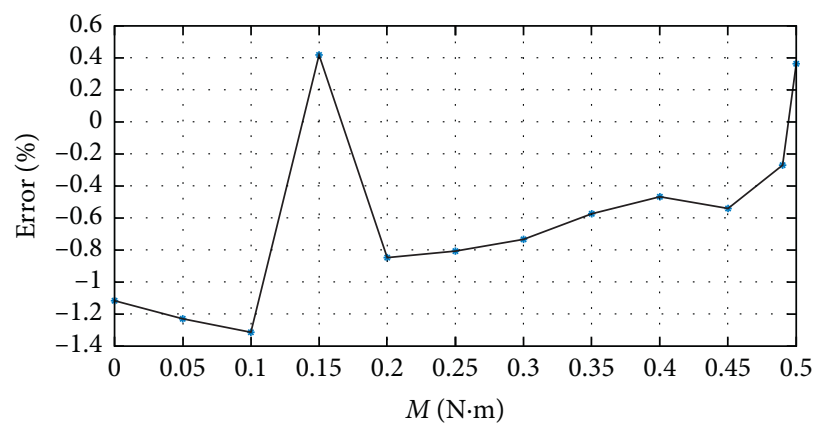

Figure 15: The errors of the rotation angles between the comprehensive solution and the nonlinear finite element results.

vertical force attains $F_{y}=-11.42 \mathrm{~N}$ and the rotation angle is $\Delta \theta=31.86^{\circ}$, for which the deflected shape agrees well with the result calculated by NFEA, as shown in Figure 18. The maximum stress of the pivot subject to $F_{y}=-11.42 \mathrm{~N}$ and $M=0.2 \mathrm{~N} \cdot \mathrm{m}$ is $\sigma_{\max }=33.995 \mathrm{MPa}$ and occurs in the deflected spring $\mathrm{O}_{2} B$ shown by the diamond shape in Figure 18 . When $M$ is greater than $0.49 \mathrm{~N} \cdot \mathrm{m}$, the pivot subject to the negative force directly leads to the failure of the spring, so in this case, the pivot can only withstand the positive vertical force.

For $M=0 \mathrm{~N} \cdot \mathrm{m}$, the pivot only subject to vertical force and the buckling of the spring may take place. For the buckled LITF pivot, the maximum bending stress $\sigma_{\max }$ may be less than the yield strength of the material used, but the LITF pivot has been invalidated, so the maximum negative vertical force for $M=0 \mathrm{~N} \cdot \mathrm{m}$ is equal to the critical buckling force.

The buckled springs can be seemed as the fixed-guided beams with two inflection points $(m=2)$ that perhaps have two deformed shapes (I) and (II), as shown in Figure 19. The vertical displacement of the freedom is $\delta$, and the end slope of the buckled spring remains constant, i.e., $\theta_{o} \equiv 0$. 


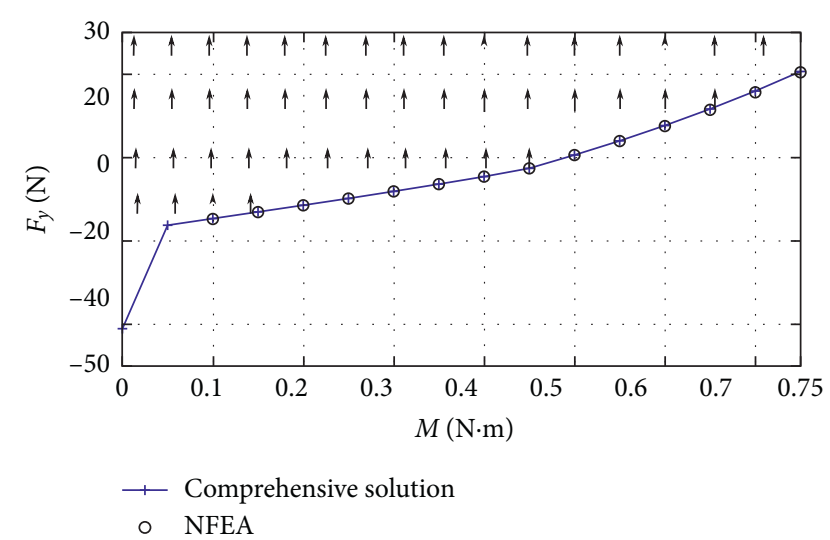

FIgURE 16: Allowable maximum vertical force of the pivot subject to different moments.

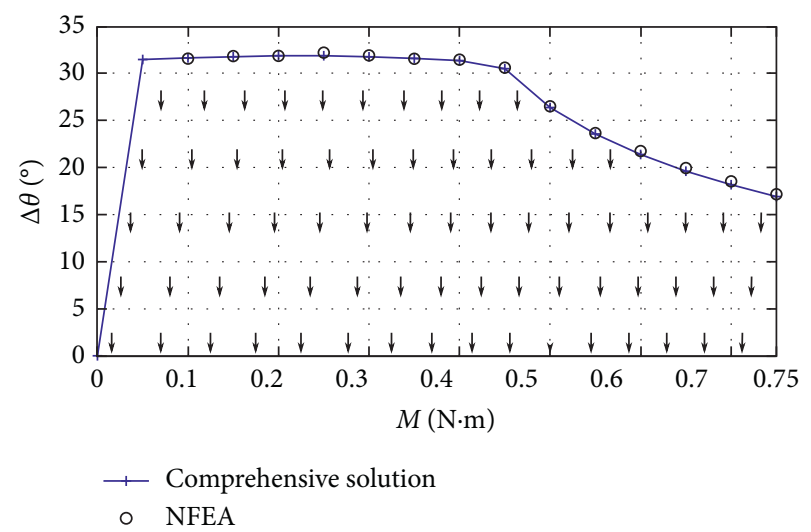

FIGURE 17: Corresponding rotation angle of the pivot subject to different moments and maximum vertical force depicted in Figure 16.

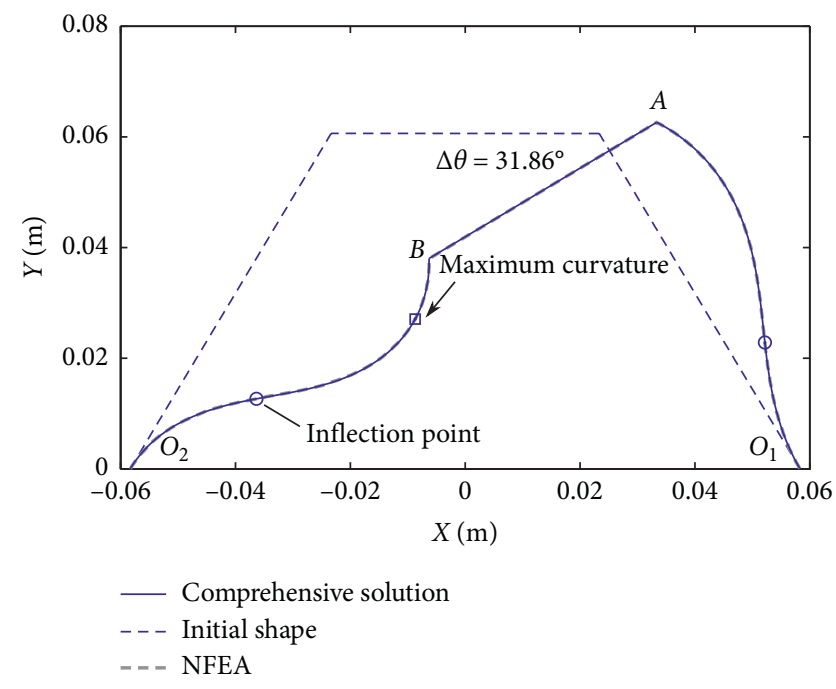

Figure 18: Deformed shape of the positive pivot subject to $F_{x}=0 \mathrm{~N}, F_{y}=-11.42 \mathrm{~N}$, and $M=0.2 \mathrm{~N} \cdot \mathrm{m}$.
For the buckled springs, the coordinates of the free end are given as

$$
\left.\begin{array}{l}
a=L-\delta \cos \beta \\
b=-\delta \sin \beta
\end{array}\right\}
$$

The vertical force $F_{y}$ can be solved as

$$
F_{y}=2 P(\sin \beta-n \cos \beta) \text {. }
$$

Substituting $m=2, \theta_{o} \equiv 0$, and equation (8) into equation (9) yields

$$
\begin{aligned}
& P=\frac{16 \mathrm{EI}}{\sqrt{1+n^{2}} L^{2}} F^{2}(t), \\
& \frac{a}{L}=-\frac{n}{\sqrt{1+n^{2}}}\left[1-\frac{2 E(t)}{F(t)}\right], \\
& \frac{b}{L}=\frac{1}{\sqrt{1+n^{2}}}\left[1-\frac{2 E(t)}{F(t)}\right] .
\end{aligned}
$$

From equations (18), (21), and (22), $n$ is

$$
n=-\frac{a}{b}=\frac{L-\delta \cos \beta}{\delta \sin \beta} \text {. }
$$

Substituting equations (20) and (23) into equation (19) yields

$$
F_{y}=\frac{32 \mathrm{EI}}{L^{2}} \frac{\delta-L \cos \beta}{\sqrt{\delta^{2}+L^{2}-2 L \delta \cos \beta}} F^{2}(t) .
$$

When $F_{y}$ reaches the critical buckling force, we have $n \longrightarrow \infty$ and $(a / L) \longrightarrow 0$, and then equation (21) reduces to $(F(t) / E(t)) \longrightarrow 1$ and has

$$
t=0 .
$$

We have $F(t)=\pi / 2$; then, the critical buckling force $F_{c r}$ from equation (24) is

$$
F_{c r}=-\frac{32 \mathrm{EI} \cos \beta}{L^{2}} F^{2}(0)=-\frac{8 \pi^{2} \mathrm{EI} \cos \beta}{L^{2}} .
$$

Thus, for $M=0$, the maximum negative vertical force of the pivot $F_{y}$ is determined by the critical buckling force solved by equation (26) and equal to $-41.0272 \mathrm{~N}$, as shown in Figure 16. When the pivot is loaded only by the vertical force, the leaf with two inflection points includes two deflection paths, which are shown in the left-hand leaf and right-hand leaf of Figure 19. The choice of the two solutions is decided by the processing factor of the leaf.

3.2. Parallelogram Flexure. A parallelogram flexure is a onedegree-of-freedom device that obtains accurate motion by the bending of the springs. Many authors have contributed to this problem; for example, Awtar et al. [18] proposed a beam constraint model and Dibiasio et al. [19] presented a pseudorigid-body model to simplify the derivation and 
calculation. In the paper, the kinetostatic model is also suitable to analyze the parallelogram flexure.

For the parameters of the mechanism given in Table 1, the leaf springs $O_{1} A$ and $O_{2} B$ with one inflection point guide the motion of link $A B$ with minimal rotation. When $F_{x}$ is applied at point $C$, the horizontal displacement $\Delta X$ is obtained to arrive at a static equilibrium state, as shown in Figure 20. With increasing horizontal force, the nonlinear characteristics of the curve are gradually obvious and the rotation angle $\Delta \theta$ is slowly increasing, as shown in Figure 21. When $F_{x}=8.5 \mathrm{~N}$ is loaded at point $C, \Delta X=0.032 \mathrm{~m}$ and $\Delta \theta=-0.145^{\circ}$, for which the maximum curvature occurring at point $\mathrm{O}_{2}$ is $K_{\max }=46.9396 \mathrm{~m}^{-1}$ and the maximum bending stress $\sigma_{\max }$ solved by equation (16) is slightly less than $S_{y}$.

The rotation angle $\Delta \theta$ of link $\mathrm{AB}$ is a parasitic error motion that is undesirable in response to the horizontal force $F_{x}$, which may be eliminated by an appropriate combination of moment $M$ or vertical force $F_{y}$ [18]. When $F_{x}$ and $M$ are loaded simultaneously at point $C$ to ensure $\Delta \theta \equiv 0$, we have, from equations (4)-(6),

$$
\frac{M}{F_{x}}=-\frac{M_{1 o}}{P_{1}} \text {. }
$$

For a parallelogram flexure because each deflected leaf spring carries one inflection point, where the resultant moment is equal to zero and the rotation angles at the fixed and free ends of each deflected leaf spring are both equal to zero, the inflection point occurs at the middle of the deflected spring, i.e., $x=a_{1} / 2$. The moment at the inflection point is

$$
\frac{a_{1}}{2} P_{1}+M_{1 o}=0
$$

Substituting equation (28) into equation (27) yields

$$
\frac{M}{F_{x}}=\frac{a_{1}}{2}=\frac{a_{2}}{2}=\frac{L+\Delta Y}{2} .
$$

For $F_{y}=0$, the ratios in equation (29) during the intermediate stage are approximatively constant, which agree well with the results of Ref. [18] equal to $0.5 \mathrm{~L}$, as shown in Figures 22 and 23. Then, the ratios between $M$ and $F_{x}$ are less than $0.5 \mathrm{~L}$ with increasing horizontal force $F_{x}$ and the corresponding transverse stiffness gradually increases for protecting $\Delta \theta \equiv 0$. As listed in Table 2, the corresponding moments and displacements for $F_{x}=2 \sim 10 \mathrm{~N}$ solved by the elliptic integral solution agrees well with the load-deflection relationship expressed in equation (29) and the deflected shapes of the pivot subject to $F_{x}$ and $M$ are shown in Figure 24.

When $F_{x}$ and $F_{y}$ are loaded at point $C$ simultaneously, $M$ is needed to ensure that $\Delta \theta \equiv 0$. In this case, the inflection points also appear in the middle of the deflected leaf springs. However, if $F_{y}$ is a tensile force, the ratios between $M$ and $F_{x}$ are less than $a_{1} / 2$ because of $n$ being less than zero. On the contrary, for $F_{y}$ as a pressure, the ratios between $M$ and $F_{x}$ are greater than $a_{1} / 2$. The more obvious nonlinearity of the

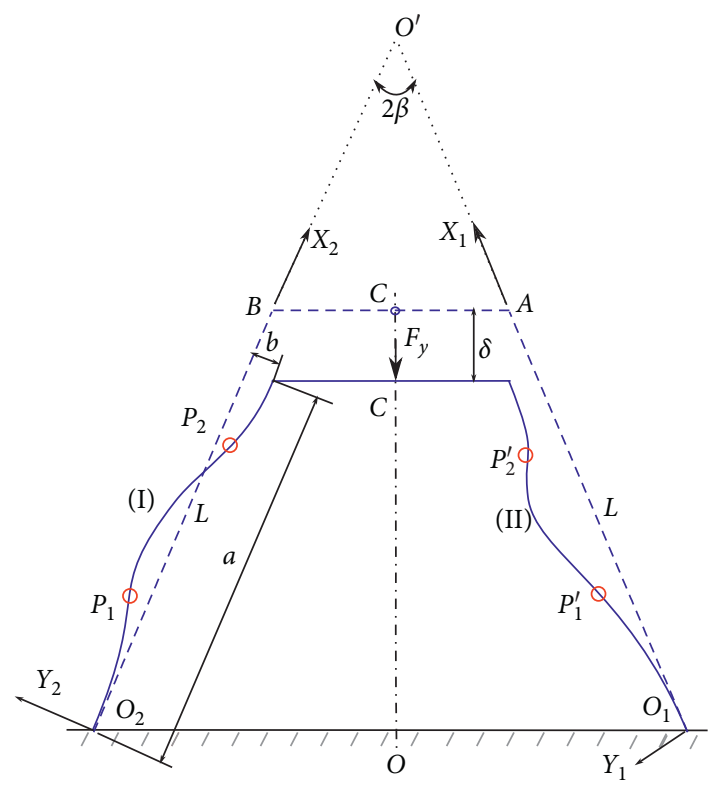

FIgURE 19: Buckling deformation of the LITF pivot.

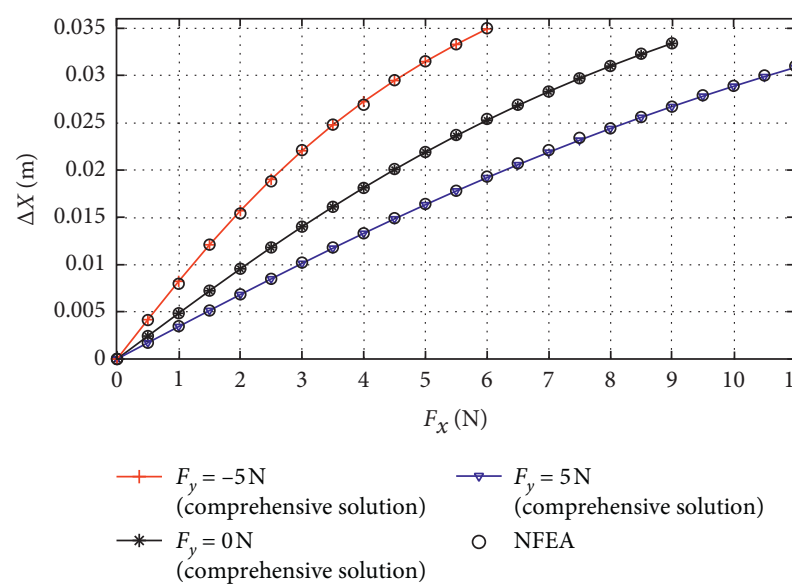

FIGURE 20: Plots of horizontal force $F_{x}$ vs horizontal displacement $\Delta X$.

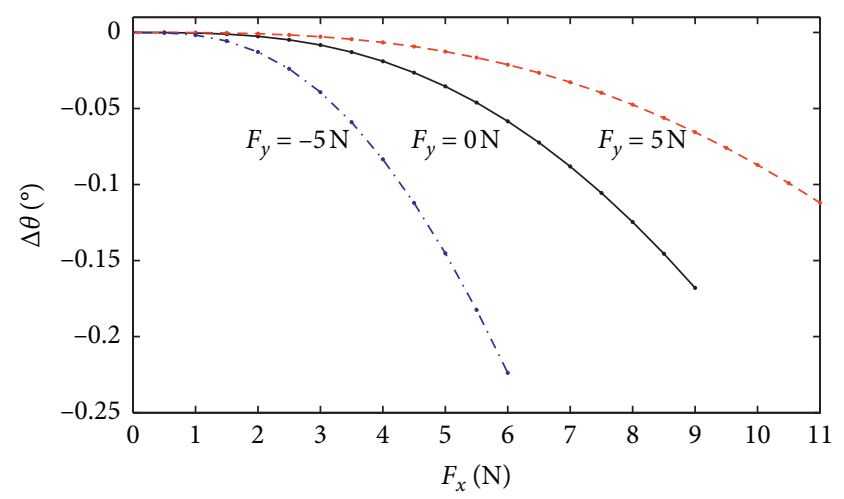

Figure 21: Plots of rotation angle $\Delta \theta$ of parallelogram flexure subject to horizontal force $F_{x}$. 


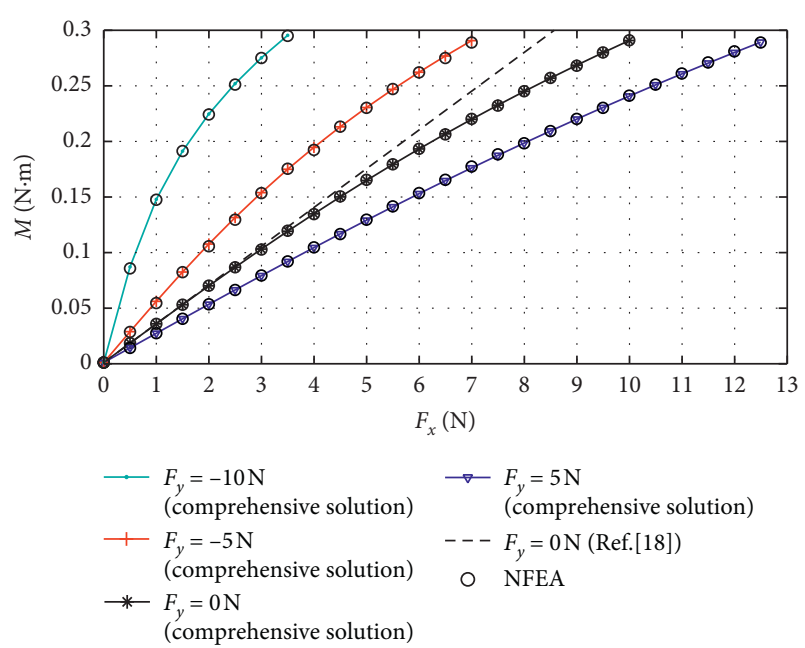

Figure 22: Moment $M$ vs $F_{x}$ for end angle $\Delta \theta \equiv 0$ of parallelogram flexure.

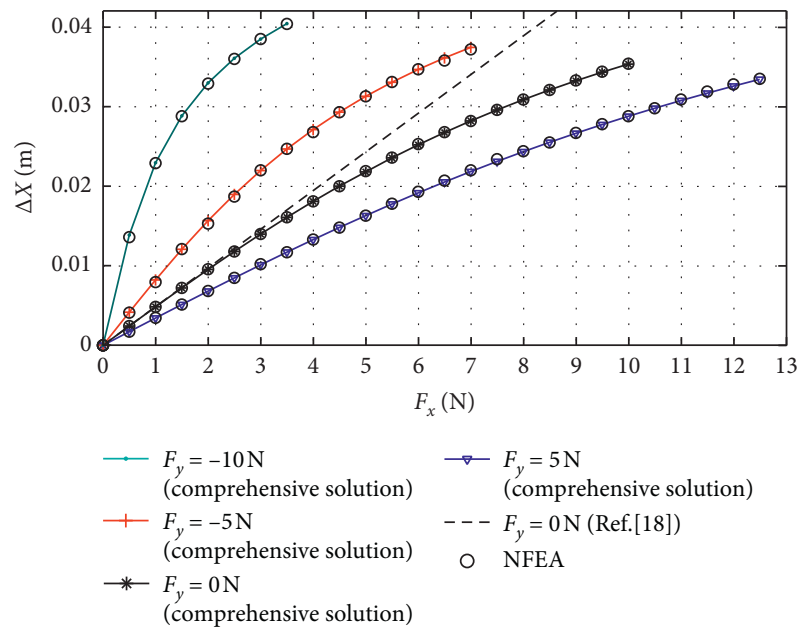

Figure 23: Horizontal displacement $\Delta_{x}$ vs $F_{x}$ for end angle $\Delta \theta \equiv 0$ of parallelogram flexure.

TABLE 2: Loads and deflections of parallelogram flexure.

\begin{tabular}{lcccc}
\hline$F_{x}(\mathrm{~N})$ & $F_{y}(\mathrm{~N})$ & $M(\mathrm{~N} \cdot \mathrm{m})$ & $\Delta X(\mathrm{~m})$ & $\Delta Y(\mathrm{~m})$ \\
\hline 2 & 0 & 0.0692 & 0.0095 & $-7.84 \times 10^{-4}$ \\
4 & 0 & 0.1343 & 0.0181 & $-2.87 \times 10^{-3}$ \\
6 & 0 & 0.1929 & 0.0252 & $-5.71 \times 10^{-3}$ \\
8 & 0 & 0.2448 & 0.0308 & -0.0088 \\
10 & 0 & 0.2908 & 0.0354 & -0.0118 \\
\hline
\end{tabular}

pivot appears with increasing pressure $F_{y}$, as shown in Figure 23. Until the pressure reaches the critical buckling force calculated by equation $(26)\left(F_{y}=-47.3741 \mathrm{~N}\right)$, the buckling of the pivot leads directly to failure.

\section{Conclusions}

The comprehensive elliptic integral solution was used for building the generalized model of LITF pivots and solving

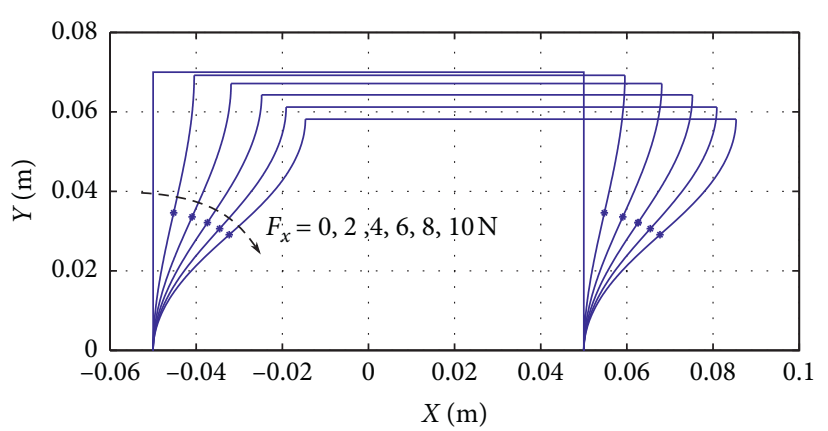

Figure 24: Deflected shapes of parallelogram flexure loaded by $F_{x}$ and $M$ for $\Delta \theta \equiv 0$.

nonlinear deflection problems. For the LITF pivot, the accurate deflected shapes are described subject to different horizontal forces, vertical forces, and moments. Furthermore, based on the strength check and the analysis of the critical buckling force, motion range and load-bearing capability for the pivot are evaluated. For the parallelogram flexure, two cases for free rotation angle and constant rotation angle are discussed. The more accurate ratio between horizontal force and moment is proposed to ensure that the rotation angle remains constant. The analytical results for the maximum rotation angle of the LITF pivot subject to horizontal force and moment solved by the comprehensive elliptic integral solution are within 1.5 percent error compared to the finite element analysis results.

\section{Data Availability}

The calculation data used to support the findings of this study are available from the corresponding author upon request.

\section{Conflicts of Interest}

The authors declare that they have no conflicts of interest.

\section{Acknowledgments}

The authors gratefully acknowledge financial support from the National Natural Science Foundation of China under Grant nos. 51605359 and 51805396.

\section{References}

[1] S. T. Smith, Flexures: Elements of Elastic Mechanisms, CRC Press, Boca Raton, FL, USA, 2000.

[2] B. P. Trease, Y.-M. Moon, and S. Kota, "Design of largedisplacement compliant joints," Journal of Mechanical Design, vol. 127, no. 4, pp. 788-798, 2005.

[3] L. J. Lai, G. Y. Gu, H. Zhou et al., "Design and analysis of a spatial Remote center of compliance mechanism," in Intelligent Robotics and Applications, pp. 385-396, Springer, Berlin, Germany, 2013.

[4] S. Henein, P. Spanoudakis, S. Droz et al., "Flexure pivot for aerospace mechanisms," in Proceedings of the 10th European Space Mechanisms and Tribology Symposium, San Sebastian, Spain, 2003. 
[5] G. Chen, X. Liu, H. Gao, and J. Jia, "A generalized model for conic flexure hinges," Review of Scientific Instruments, vol. 80, no. 5, Article ID 055106, 2009.

[6] G. Chen, X. Liu, and Y. Du, "Elliptical-arc-fillet flexure hinges: towards a generalized model for most commonly used flexure hinges," Journal of Mechanical Design, vol. 133, no. 8, Article ID 081002, 2011.

[7] N. Lobontiu, Compliant Mechanisms: Design of Flexure Hinges, CRC Press, Boca Raton, FL, USA, 2010.

[8] A. Midha, S. G. Bapat, A. Mavanthoor, and V. Chinta, "Analysis of a fixed-guided compliant beam with an inflection point using the pseudo-rigid-body model (PRMB) concept," in Proceedings of the ASME 2012 International Design Engineering Technical Conferences \& Computers and Information in Engineering Conference, Chicago, IL, USA, August 2012.

[9] N. Ciblak and H. Lipkin, "Design and analysis of remote center of compliance structures," Journal of Robotic Systems, vol. 20, no. 8, pp. 415-427, 2003.

[10] X. Pei, J. Yu, G. Zong, and S. Bi, "The stiffness model of leaftype isosceles trapezoidal flexural pivots," ASME Journal of Mechanical Design, vol. 130, no. 8, Article ID 082303, 2008.

[11] H. Zhao, S. Bi, and J. Yu, "Nonlinear deformation behavior of a beam-BASED flexural pivot with monolithic arrangement," Precision Engineering, vol. 35, no. 2, pp. 369-382, 2011.

[12] A. N. Polilov, N. A. Tatus', and X. Tian, "Analysis of efficiency of uniform-strength composite leaf springs under various loading conditions," Journal of Machinery Manufacture and Reliability, vol. 48, no. 5, pp. 431-439, 2019.

[13] L. L. Howell, Compliant Mechanisms, Wiley-Interscience, New York, NY, USA, 2001.

[14] C. Kimball and L.-W. Tsai, "Modeling of flexural beams subjected to arbitrary end loads," Journal of Mechanical Design, vol. 124, no. 2, pp. 223-235, 2002.

[15] A. Zhang and G. Chen, "A comprehensive elliptic integral solution to the large deflection problems of thin beams in compliant mechanisms," Journal of Mechanism and Robotics, vol. 5, no. 2, Article ID 021006, 2013.

[16] P. F. Byrd and M. D. Friedman, Handbook of Elliptic Integrals for Engineers and Physicists, Springer-Verlag, Berlin, Germany, 1954.

[17] F. P. Beer, E. R. Johnston Jr., J. DeWolf, and D. Mazurek, Mechanics of Materials, McGraw-Hill, New York, NY, USA, 2012.

[18] S. Awtar, A. H. Slocum, and E. Sevincer, "Characteristics of beam-based flexure modules," Journal of Mechanical Design, vol. 129, no. 6, pp. 625-639, 2007.

[19] C. M. Dibiasio, L. L. Howell, S. P. Magleby et al., "Comparison of molecular simulation and pseudo-rigid-body model predictions for a carbon nanotube-based compliant parallelguiding mechanism," Journal of Mechanical Design, vol. 130, no. 4, Article ID 042308, 2008. 